\title{
FACE RECOGNITION USING 3D SUMMATION INVARIANT FEATURES
}

\author{
Wei-Yang Lin, Kin-Chung wong, Yu Hen Hu, and Nigel Boston* \\ University of Wisconsin-Madison \\ Department of Electrical and Computer Engineering \\ 1415 Engineering Drive, Madison WI, 53706 USA \\ weiyanglin@wisc.edu
}

\begin{abstract}
In this paper, we developed a family of $2 \mathrm{D}$ and $3 \mathrm{D}$ invariant features with applications to 3D human faces recognition. The main contributions of this paper are: (a) systematically deriving a family of novel features, called summation invariant that are invariant to Euclidean transformation in both 2D and 3D; (b) developing an effective method to apply summation invariant to the 3D face recognition problem. Tested with the 3D data from the Face Recognition Grand Challenge v1.0 dataset, the proposed new features exhibit achieves a performance that rivals the best $3 \mathrm{D}$ face recognition algorithms reported so far.
\end{abstract}

\section{INTRODUCTION}

Human face recognition has received unprecedented interest in recent years [21]. However, a majority of current face recognition systems are designed for $2 \mathrm{D}$ facial images. There are much fewer works on $3 \mathrm{D}$ face recognition. Among those reported $3 \mathrm{D}$ face recognition algorithms, there are three main approaches:

The first approach [13], [10] regards a 3D facial range image as a gray-level image and apply standard 2D algorithm such as eigen-face to perform the classification task. The second approach [10] is to apply a deformable 3D facial surface model. The third approach [3], [19] is to extract features from a $3 \mathrm{D}$ facial surface that are invariant to pose variations. These approaches focus on the classical differential invariants such as Gaussian curvature.

A fundamental weakness of differential invariants is that the high-order derivative operations are sensitive to noisy data. Hann and Hickman proposed a new family of integral invariant [7] that are based on integration rather than derivative operators. However, since their formulation is defined on continuous functions, when applied to sampled data, such as digital images, it suffers from excessive numerical errors. Several related approaches such as the semi-differential invariants [14], and affine quasi-invariant arc-length [18] are also

\footnotetext{
* The authors have been partially supported by the National Science Foundation under Grant No. CCF-0434355.
}

defined on continuous functions and hence share the same numerical concerns as the integral invariants.

Recently, we proposed a new family of invariants, called summation invariant [12] that is based on summation of discretized sample data. As such, these invariants are likely to be more robust to noise and numerical errors. Based on this preliminary result, in this paper, we developed a new family of summation invariants in both $\mathbb{R}^{2}$ and $\mathbb{R}^{3}$ with respect to the Eucledian transformation (rotation and translation). Moreover, we developed an efficient approach to extract these 2D and $3 \mathrm{D}$ summation invariants from a given $3 \mathrm{D}$ range image of human face and demonstrated superior performance. We have conducted extensive experiments using the Face Recognition Grand Challenge v1.0 dataset and the BEE (Biometric Experimentation Environment) package. Our method yields the best performance that has been reported so far [10].

The rest of this paper is organized as follows. Section 2 describes the summation invariant for the Euclidean transformation group. Both $2 \mathrm{D}$ and $3 \mathrm{D}$ cases are discussed. The application to $3 \mathrm{D}$ face recognition is presented in section 3 , with experimental results summarized in section 5 .

\section{SUMMATION INVARIANTS}

We employ the method of moving frames [4] by Élie Cartan, to systematically construct mathematical invariants under group actions. For 3D face recognition, we seek features that are invariant to pose variations which can be modeled by Euclidean geometrical transformations (rotation and translation). Our approach is similar to that of our early work [12] for the affine transformation group acting on $\mathbb{R}^{2}$.

\subsection{Euclidean summation invariants of curves}

Denote $(x[n], y[n])$ and $(\bar{x}[n], \bar{y}[n]) 0 \leq n \leq N-1$ respectively to be the corresponding point on a curve in $\mathbb{R}^{2}$ before and after an Euclidean transformation

$$
\left[\begin{array}{c}
\bar{x}[n] \\
\bar{y}[n]
\end{array}\right]=\left[\begin{array}{cc}
\cos (\theta) & -\sin (\theta) \\
\sin (\theta) & \cos (\theta)
\end{array}\right]\left[\begin{array}{c}
x[n] \\
y[n]
\end{array}\right]+\left[\begin{array}{l}
a \\
b
\end{array}\right]
$$


where $a, b, \theta \in \mathbb{R}$ are parameters. Define the $i, j^{\text {th }}$ potentials of this curve as

$$
P_{i, j}=\sum_{n=0}^{N-1} x^{i}[n] \cdot y^{j}[n]
$$

where $i, j$ are nonnegative integers, $i+j=k$ and $k \neq 0$. Similar, we denote $\bar{P}_{i, j}$ to be the potentials after Eclidean transformation.

To systematically derive a family of invariants with respct to the Euclidean transform group, our approach is as follows:

1. Construct a prolonged potential jet space $\left(\bar{x}_{0}, \bar{y}_{0}, \bar{x}_{1}\right.$, $\left.\bar{y}_{1}, \bar{P}^{i, j}\right)$ where $\bar{x}_{0}=\bar{x}[0], \bar{y}_{0}=\bar{y}[0], \bar{x}_{1}=\bar{x}[N-1]$, $\bar{y}_{0}=\bar{y}[N-1]$.

2. Solve for parameters (called moving frame $a, b$, and $\theta$ of the Euclidean transform from a set of a normalization equations:

$$
\left(\bar{x}_{0}, \bar{y}_{0}, \bar{y}_{1}\right)=(0,0,0)
$$

3. Express the analytical expression of the solution in terms of $x_{0}, y_{0}$, and potentials. Substitute these solutions into a transformed potential $\bar{P}_{i, j}=\sum_{n=0}^{N-1} \bar{x}^{i}[n] \bar{y}^{j}[n]$. The result is the desired invariant $\eta_{i, j}$

Note that we are free to specify normalization equations as long as they admit explicit analytical solution, i.e.a moving frame can be found. We have derived all five first and second order invariant functions, $i+j=1$ or $2, \eta_{1,0}, \eta_{0,1}, \eta_{2,0}, \eta_{1,1}$, and $\eta_{0,2}$. Due to space limitation, we only list the first two here:

$$
\begin{aligned}
\eta_{1,0}= & \left(x_{1}-x_{0}\right) P_{1,0}+\left(y_{1}-y_{0}\right) P_{0,1}+N x_{0}\left(x_{0}-x_{1}\right) \\
& +N y_{0}\left(y_{0}-y_{1}\right) \\
\eta_{0,1}= & \left(y_{1}-y_{0}\right) P_{1,0}+\left(x_{0}-x_{1}\right) P_{0,1}+N\left(x_{1} y_{0}-x_{0} y_{1}\right)
\end{aligned}
$$

\subsection{Euclidean summation invariants of surfaces}

Above procedure can readily be generalized to a surface in $\mathbb{R}^{3}$. Albeit, some complications must be overcome. Given a parameterized surface $(x[m, n], y[m, n], z[m, n])$ with $m=$ $0, \ldots, M-1$ and $n=0, \ldots, N-1$, its corresponding $p o$ tentials is defined by

$$
Q_{i, j, k}=\sum_{m=0}^{M-1} \sum_{n=0}^{N-1} x^{i}[m, n] \cdot y^{j}[m, n] \cdot z^{k}[m, n]
$$

where $i, j, k$ are nonnegative integers, $i+j+k=\ell$ and $\ell \neq 0$.

In $3 \mathrm{D}$, the Euclidean transformation is parameterized by 3 elementary rotation matrices $\mathbf{R}_{1}, \mathbf{R}_{2}, \mathbf{R}_{3}$, and a 3D translation vector $\mathbf{T}$. This increases the number of parameters to

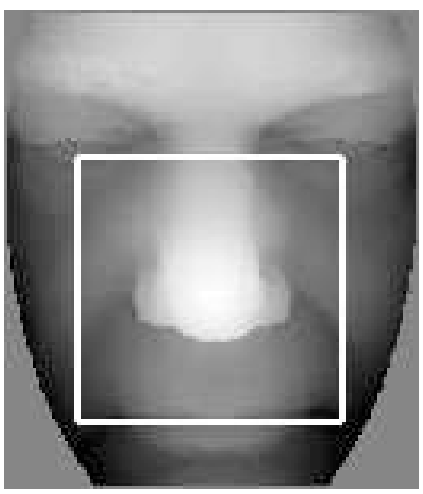

Fig. 1. Normalized range image and an $81 \times 81$ region centered at the nose tip.

six. These parameters are to be solved from a set of six normailzation equations:

$$
\begin{aligned}
(\bar{x}[0,0], \bar{y}[0,0], \bar{z}[0,0], \bar{y}[M-1,0], \bar{z}[M-1,0], \\
\bar{z}[0, N-1])=(0,0,0,0,0,0)
\end{aligned}
$$

The first three equations yields $\mathbf{T}=[x[0,0], y[0,0], z[0,0]]^{T}$. In order to satisfy the next two equations, denote $\mathbf{A}=$ $[x[M-1,0], y[M-1,0], z[M-1,0]]^{T}$ and compute its polor coordinates $\left(R_{a}, \theta_{a}, \phi_{a}\right)$. If two of the elementary matrics are defined on $\theta_{a}$, and $\phi_{a}$ respectively, then it is guaranteed that $\bar{y}[M-1,0]=0$, and $\bar{z}[M-1,0]=0$. Thus, the fourth and fifth normalized equations are solved.

Finally, to solve the last equation, denote $\mathbf{B}=\mathbf{R}_{2} \mathbf{R}_{1}$. $\left([x[0, N-1] \quad y[0, N-1] \quad z[0, N-1]]^{T}-\mathbf{T}\right)$. Again by representing $\mathbf{B}$ in polar coordinates, $\left(R_{c}, \theta_{c}, \phi_{c}\right)$, one may determine $\mathbf{R}_{3}$ using $\phi_{c}$. By substituting these solutions into the transformed potentials $\bar{Q}_{i, j, k}$, we obtain desired invariants $\kappa_{i, j, k}$.

\section{APPLICATION TO FACE RECOGNITION}

We use the 3D facial images distributed with the Face Recognition Grand Challenge (FRGC) dataset [15] to conduct face recognition experiments. The $3 \mathrm{D}$ data contains 275 subjects ( 1 to 8 range scans per subject) and a total of 943 range scans. Each range scan has a resolution of $640 \times 480$ pixels.

Our experiment procedures closely follow those defined for the baseline algorithm provided by FRGC. We extract invariant features from an $N \times N(N=81)$ rectangular region centered at the nose tip of the $3 \mathrm{D}$ facial map. An example is shown in Figure 1. The positions of the nose tips are manually selected and are provided by the FRGC dataset.

For 2D invariants $\eta_{i, j}$, facial range data are resampled uniformly with respect to arc-length. Specifically, for each row on a range data, we first compute its arc-length and resample 


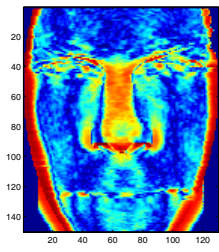

(a)

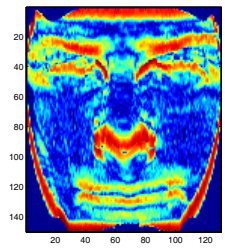

(b)
Fig. 2. For each range image, we compute summation invariants at each pixel: (a) Summation invariant is computed from a $1 \times L$ horizontal window and (b) from a $L \times 1$ vertical window.

it uniformly with respect to arc-length. Then, we perform the same resampling on each column. For surface invariants $\kappa_{i, j}$, we do not perform resampling on the normalized range data.

We extract a semi-local summation invariant from each row and each column of the $81 \times 81$ rectangular region and use the results as invariant features. For curve invariants $\eta_{i, j}$, we compute their semi-local summation invariants from a local window. At each pixel, semi-local summation invariants are computed both vertically and horizontally. The length of local window is chosen to be $L=21$. Similarly, we also compute semi-local summation invariants $\kappa_{i, j, k}$ from a local window surrounding each pixel. The window size for surface invariants is $17 \times 17$.

In order to reduce the size of the feature vectors, we use principal component analysis (PCA) to compute their subspace projections. The PCA basis is computed using the training set (183 range images) specified by the experiment 3 of the FRGC v1.0. The classification is accomplished by using subspace projections. The Mahalanobis cosine distance metric is used in these experiments.

The code is implemented in non-optimized $\mathrm{C}$ language. The experiments are conducted under Linux operation system with $3.40 \mathrm{GHz}$ XEON processor and $2 \mathrm{~GB}$ memory.

\section{EXPERIMENTAL RESULTS}

We have conducted a series of experiments to assess the performance of the proposed algorithm. Due to space limitation, we only report the final results.

\subsection{Effects of combining summation invariants}

The purpose of this experiment is to investigate how to best combine several different summation invariant to achieve highest performance. Only summation invariants that give higher performance individually are combined. Figure 3 shows the ROC curves of combining two summation invariants. The fusion strategy is simply adding the similarity scores from two different summation invariants. We observe that fusion does

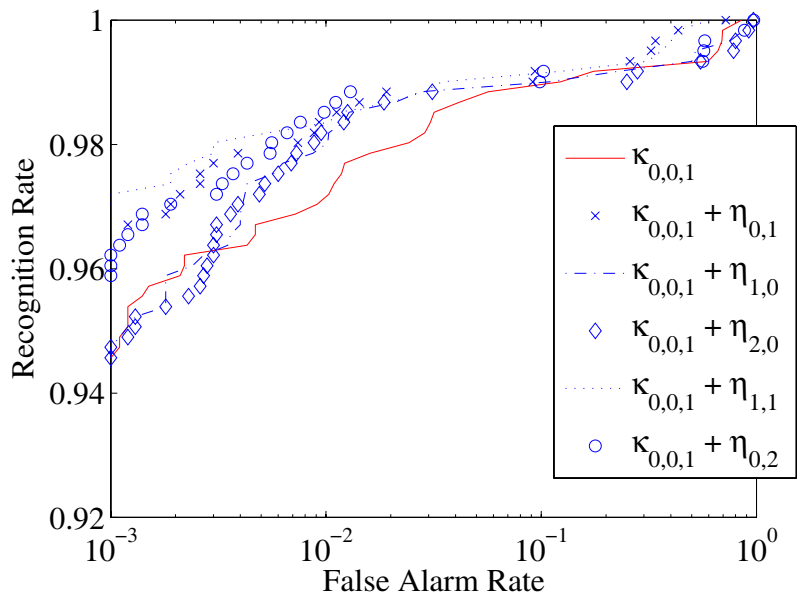

(b)

Fig. 3. ROC performance obtained by combining $\kappa_{0,0,1}$ and others.

not always yields higher recognition rate than that of a single summation invariant. However, at low false alarm rate, eg. $0.1 \%$, the improvement provided by fusion two or more summation invariant is quite significant.

\subsection{Comparison with other $3 D$ face recognition algorithms}

In this section, we conduct three experiments to evaluate the performance of our algorithm and the FRGC baseline algorithm. In the first, we simply run the FRGC baseline algorithm on 3D data alone. In the second, we still run the FRGC baseline algorithm on 3D data but using only the cropped region rather than the whole normalized range data. In Fig 4, the second experiment shows a lower recognition rate than the first one. This is reasonable because the second experiment uses less data to perform recognition. In the third experiment, we compute $\eta_{1,1}$ and $\kappa_{0,0,1}$ from the cropped region. Our algorithm yields the highest recognition rate as one can see in Fig 4 (At $0.1 \%$ false alarm rate, the recognition rate is $97.2 \%$ ). The results clearly indicate that summation invariants offer statistically significant better recognition performance than the range data itself. Except for the FRGC 3D baseline, Kakadiaris etal.[10] also apply their algorithm to the range images from FRGC v1.0 dataset and report about $97.0 \%$ recognition rate at $0.1 \%$ false alarm rate. So, the proposed method has the same recognition performamce as the the best results ever published.

\section{DISCUSSION AND CONCLUSION}

The value of summation invariants in the context of $3 \mathrm{D}$ face recognition is evaluated in this paper. We extract geometric features of facial surfaces using summation invariants and 


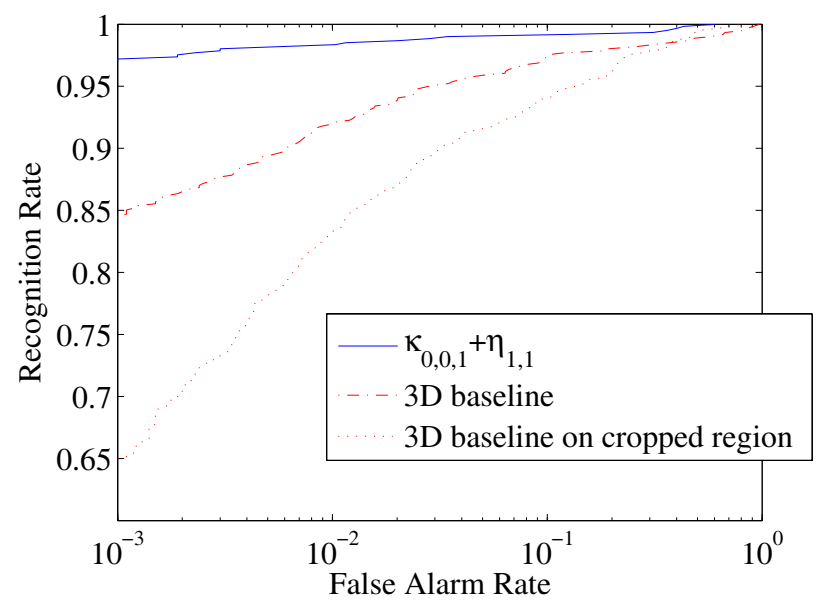

Fig. 4. Comparison with FRGC 3D baseline algorithm. We apply the FRGC 3D baseline algorithm on the normalized depth map and the cropped region shown in Fig 1. Their corresponding ROC curves are shown by the solid line and the dash line respectively.

apply PCA on the resulting representation. Our results compares favorably to those reported by Kakadiaris etal.[10]. We are currently working on FRGC version 2.0 dataset, and explore additional $2 \mathrm{D}$ and $3 \mathrm{D}$ feature extraction method to improve the $3 \mathrm{D}$ face recogntion performance.

\section{REFERENCES}

[1] K. W. Bowyer, K. Chang, and P. Flynn. A survey of approaches to three-dimensional face recognition. In Proceedings of International Conference on Pattern Recognition, volume 1, pages 358-61, 2004.

[2] A. M. Bronstein, M. M. Bronstein, and R. Kimmel. Expression-invariant 3d face recognition. In Proceedings of the 4th International Conference on Audio- and Video-Based Biometric Person Authentication, pages 62-9, 2003.

[3] J. Y. Cartoux, J. T. Lapreste, and M. Richetin. Face authentification or recognition by profile extraction from range images. In Proceedings of Workshop on Interpretation of $3 D$ Scenes, pages 194-9, 1989.

[4] M. Fels and P. J. Olver. Moving coframes: I. a practical algorithm. Acta Applicandae Mathematicae, 51(2):161 - 213, 1998.

[5] M. Fels and P. J. Olver. Moving coframes: Ii. regularization and theoretical foundations. Acta Applicandae Mathematicae, 55(2): 127 - 208, 1999.

[6] G. G. Gordon. Face recognition based on depth and curvature features. In Proceedings of IEEE Computer Society Conference on Computer Vision and Pattern Recognition, pages 80810, 1992.

[7] C. E. Hann and M. S. Hickman. Projective curvature and integral invariants. Acta Applicandae Mathematicae, 74(2):177193, 2002.
[8] T. Heseltine, N. Pears, and J. Austin. Three-dimensional face recognition: An eigensurface approach. In Proceedings of the International Conference on Image Processing, volume 5, pages 1421-1424, 2004.

[9] C. Hesher, A. Srivastava, and G. Erlebacher. A novel technique for face recognition using range imaging. In Proceedings of the Seventh International Symposium on Signal Processing and its Applications, volume vol.2, pages 201-4, 2003.

[10] I. A. Kakadiaris, G. Passalis, T. Theoharis, G. Toderici, I. Konstantinidis, and N. Murtuza. Multimodal face recognition: Combination of geometry with physiological information. In Proceedings of IEEE Computer Society Conference on Computer Vision and Pattern Recognition, volume 2, pages 1022 1029, 2005.

[11] J. C. Lee and E. Milios. Matching range images of human faces. In Proceedings of Third International Conference on Computer Vision, pages 722-6, 1990.

[12] W. Y. Lin, N. Boston, and Y. H. Hu. Summation invariant and its application to shape recognition. In Proceedings of ICASSP, volume V, pages 205-208, 2005.

[13] G. Medioni and R. Waupotitsch. Face modeling and recognition in 3-d. In 2003 IEEE International Workshop on Analysis and Modeling of Faces and Gestures, pages 232-3, 2003.

[14] T. Moons, E. J. Pauwels, L. J. V. Gool, and A. Oosterlinck. Foundations of semi-differential invariants. International Journal of Computer Vision, 14(1):25-47, 1995.

[15] P. J. Phillips, P. J. Flynn, T. Scruggs, K. W. Bowyer, J. Chang, K. Hoffman, J. Marques, J. Min, and W. Worek. Overview of the face recognition grand challenge. In Proceedings of IEEE Computer Society Conference on Computer Vision and Pattern Recognition, volume 1, pages 947-54, 2005.

[16] P. J. Phillips, P. Grother, R. Micheals, D. M. Blackburn, E. Tabassi, and M. Bone. Face recognition vendor test 2002. In International Workshop on Analysis and Modeling of Faces and Gestures, page 44, 2003.

[17] P. J. Phillips, H. Moon, S. A. Rizvi, and P. J. Rauss. The feret evaluation methodology for face-recognition algorithms. IEEE Transactions on Pattern Analysis and Machine Intelligence, 22(10):1090-104, 2000.

[18] J. Sato and R. Cipolla. Affine integral invariants and matching of curves. In Proceedings of 13th International Conference on Pattern Recognition, volume 1, pages 915-19, Vienna, Austria, 1996.

[19] H. T. Tanaka, M. Ikeda, and H. Chiaki. Curvature-based face surface recognition using spherical correlation. principal directions for curved object recognition. In Proceedings of Third IEEE International Conference on Automatic Face and Gesture Recognition, pages 372-7, 1998.

[20] L. Van Gool, P. Kempenaers, and A. Oosterlinck. Recognition and semi-differential invariants. In Proceedings of IEEE Computer Society Conference on Computer Vision and Pattern Recognition, pages 454-60, 1991.

[21] W. Zhao, R. Chellappa, P. J. Phillips, and A. Rosenfeld. Face recognition: A literature survey. ACM Computing Surveys, 35(4):399-458, 2003. 